\title{
Psychiatric genetics - Does diagnosis matter?
}

\author{
Martin Alda, MD
}

\section{How specific is the role of genes in psychiatry?}

Since its beginnings, psychiatric genetics has stimulated the field of psychiatry and led to crucial questions about the nature of psychiatric disorders. An example of the early debates is the nature-nurture controversy. More recently, we have been witnessing discussions about the association between the genetic architecture of psychiatric disorders and their clinical manifestations - in other words, how are genetic mechanisms linked to behaviour, cognitive functions, and emotional regulation.

Most psychiatric disorders are heritable; many, including schizophrenia, autism and bipolar disorder, are in fact highly heritable, with heritability estimates exceeding $50 \%$. The heritability data are derived mainly from twin studies, with added support from adoption studies, showing that family resemblance is primarily due to shared genes rather than shared environment. These findings have led to an expectation that clarifying the genetic mechanisms and discoveries of specific genetic variants would lead to deeper understanding of the pathophysiology of psychiatric disorders and provide clinically useful guidance for differential diagnosis, treatment selection and/or novel treatments based on genetic mechanisms.

Until recently, specific genetic variants predisposing individuals to these disorders have been hard to pinpoint. However, modern genetic methods and largescale collaborations have led to a continuously increasing number of replicated findings. Needless to say, genetic studies are finding what their methods are supposed to find. There are a handful of loci of major effect, including copy number variations, playing a role in a minute fraction of cases (families) as well as a number of loci that are relatively common and have small effects. The latter have been identified through genome-wide association studies, and their effect sizes (relative risk values) are typically around 1.1 or less. Discoveries of such associations will, no doubt, continue with increasing sample sizes. For instance, there have been 155 reported loci associated with the risk of schizophrenia in combined samples totaling 60995 and 19 reported loci for bipolar disorder in samples of 20352 cases (unpublished data, Sullivan and colleagues, 2017).

The search for nongenetic, environmental risk factors is also relevant, but arguably even more difficult as their potential number is almost infinite. Inferring from quantitative genetic analyses, the overall nongenetic contribution to most severe psychiatric disorders is relatively small and may depend on gene $\times$ environment interactions, possibly necessitating even larger sample sizes than purely genetic studies.

The complex nature of the genetic architecture of behavioural disorders is hardly surprising. Many other traits that originally seemed genetically simple show much complexity characterized by heterogeneity, multiple mutation of varying biological impact, and gene $x$ gene or gene $x$ environment interactions. On the other hand, many less complex and more uncommon conditions can lead to behavioural phenotypes presenting, for instance, as schizophrenia. ${ }^{1}$

The large case-control samples available also permit tests of shared predisposition to disorders traditionally considered to be distinct diagnostic entities. ${ }^{2,3}$ These studies show considerable overlap in polygenic susceptibility, with reported correlations between liabilities for major psychiatric disorders in the range of 0.3 to $0.7 .^{3}$

Taken together, genetic findings in psychiatry seem to indicate that the genetic predisposition to most disorders is polygenic, pleiotropic and, to a large extent, nonspecific. An intuitive generalization of this concept is the "omnigenic" model proposed by Boyle and colleagues. ${ }^{4}$ The model postulates that many complex traits are associated with variants in "most genes expressed in diseaserelevant cells." The susceptibility to a disorder is conferred by a dysfunction in gene regulatory networks rather than changes in a single or a few coding variants. The support for the model comes from several observations that include only modest enrichment of positive

Correspondence: M. Alda, Department of Psychiatry, Dalhousie University, 5909 Veterans' Memorial Lane, Halifax, NS B3H 2E2; malda@dal.ca

DOI: $10.1503 /$ jpn. 170146 
findings for genes in what could be hypothesized as disease-related categories; numbers of positive findings in different functional categories tend to increase proportionally with the overall number of genes in each category, and the associations are spread relatively evenly throughout transcriptionally active genomic regions (e.g., more than $70 \%$ of 1 -megabase regions in the genome are known to harbor at least 1 susceptibility variant for schizophrenia ${ }^{5}$.

\section{What explains the lack of specificity?}

The current concept of largely nonspecific polygenic susceptibility underlying most psychiatric disorders has to be confronted with a host of multiple factors that complicate psychiatric research in general. The first factor to consider is the low reliability of many psychiatric diagnoses, especially in the more recent DSM-5. ${ }^{6}$ The second factor is the temporary instability of many diagnoses, even in relatively short periods of observation..$^{7}$ And in certain cases a conversion from one diagnosis to another represents a natural trajectory of the underlying illness - the most obvious example is the conversion from unipolar depression to bipolar disorder.

Yet another point is that of comorbidity. It appears that at least a proportion of the family history link between disorders could be due to comorbidity in probands, which drives the increased rates of the (comorbid) condition among relatives. This seems to have been the case, for instance, in the population-wide family history study of bipolar disorder in Sweden. ${ }^{8}$ Here, the relative risks of various psychiatric conditions were increased among relatives of bipolar probands, but their values were lower than the relative risks for comorbidities in probands.

Twin studies of major psychiatric disorders show concordance for specific diagnoses - thus, for instance, monozygotic pairs in which one twin has schizophrenia and the other has bipolar disorder exist, but these are less frequent than the shared etiology model would argue. In a study by Cardno and colleagues, ${ }^{9}$ monozygotic pairs in which one member had mania whereas the other had schizophrenia were much less common than homotypic pairs in which both members had mania or in which both members had schizophrenia. ${ }^{9}$

Nonrandom, assortative mating is also relatively common in psychiatry, and yet its impact on the genetic architecture is not fully understood. ${ }^{10}$

Clinical staging may also blur differential diagnosis. Early on, the majority of individuals with severe psychiatric disorders present with nonspecific symptoms that are difficult to differentiate cross-sectionally (although the disorders may be possible to diagnose more accurately with the help of family history and longitudinal clinical course $\left.{ }^{11}\right)$. And at late stages, the clinical picture is again confounded by the impact of repeated recurrences / relapses, iatrogenic effects of treatments, medical comorbidities, inflammatory changes, substance abuse and other factors.
An important aspect to consider is the nature of the largest genetic studies to date, carried out by the Psychiatric Genomics Consortium. Their primary, although not exclusive, focus has been genome-wide association studies. Especially in the beginning, the Consortium focused on assembling large samples in order to achieve sufficient statistical power, with less emphasis on detailed phenotyping. It is in the nature of genome-wide association studies that they are best suited for finding common variants that, for statistical and epidemiological reasons, are constrained with respect to the magnitude of their effects. However, it can be shown that analyzing heterogeneous samples together will substantially diminish the effect sizes of associations. ${ }^{12}$ Is it possible that some of the current genome-wide association study results have in reality larger effects, but that those effects come from a minority of patients?

With the above points in mind, one could speculate that in psychiatry we are facing a mix of conditions. On the one hand there are disorders meeting specific criteria, as suggested by Robins and Guze. ${ }^{13}$ Such conditions could be expected to have more or less distinct pathophysiology, and their diagnosis would have good predictive value with respect to treatment selection and prognosis. On the other hand, there may be syndromes with less distinct clinical presentation that may vary over time and that have limited clinical utility as a diagnostic category.

\section{What role can genetics play in clinical psychiatry?}

The field of psychiatric genetics promises to contribute to better understanding of pathophysiology, which in turn should lead to better, more targeted treatments. Leaving aside the fact that most genetic findings do not translate instantly into pathophysiological insights, can these aims be reconciled with the current genetic findings?

It would appear that the relevance of psychiatric diagnosis in genetics is doubtful and that at present, contribution of genetics to clinical practice might remain limited to only a few specific areas. One promising area is that of pharmacogenetics, with evidence of familiality of response to at least some treatments - certain antidepressants and lithium. ${ }^{14,15}$ But the mismatch between current nosology and genetic findings should not be a reason for abandoning the search for valid and clinically useful diagnoses. Resolving the heterogeneity of psychiatric disorders will be critically important for differential diagnosis, although it will not be achieved by genetic methods alone. More detailed phenotyping and renewed interest in family-based analyses are being considered by some of the large consortia. One promising direction is adding longitudinal dimensions to descriptive psychopathology. For instance, in case of bipolar disorder, there is indication that the characteristics of clinical course are highly familial between parents and their affected children and correlate strongly with the parental response to treatment. ${ }^{11}$ Most importantly, these are hypotheses that can be further tested in long-term prospective studies. 


\section{Conclusion}

Genetic research has not yet led to major changes in psychiatric practice. At the same time, it has played an important role in highlighting the complexity of psychiatric disorders and in challenging current psychiatric nosology.

Affiliations: From the Department of Psychiatry, Dalhousie University, Halifax, NS, Canada.

Competing interests: None declared.

\section{References}

1. Propping P. Genetic disorders presenting as "schizophrenia". Karl Bonhoeffer's early view of the psychoses in the light of medical genetics. Hum Genet 1983;65:1-10.

2. Cross-disorder group of the Psychiatric Genomics Consortium. Identification of risk loci with shared effects on five major psychiatric disorders: a genome-wide analysis. Lancet 2013;20:1371-9.

3. Lee SH, Ripke S, Neale BM, et al. Genetic relationship between five psychiatric disorders estimated from genome-wide SNPs. Nat Genet 2013;45:984-94.

4. Boyle EA, Li YI, Pritchard JK. An expanded view of complex traits: from polygenic to omnigenic. Cell 2017;169:1177-86.

5. Visscher PM, Wray NR, Zhang Q, et al. 10 years of GWAS discovery: biology, function, and translation. Am J Hum Genet 2017; 101:5-22.
6. Regier DA, Narrow WE, Clarke DE, et al. DSM- 5 field trials in the United States and Canada, part II: test-retest reliability of selected categorical diagnoses. Am J Psychiatry 2013;170:59-70.

7. Schwartz JE, Fennig S, Tanenberg-Karant M, et al. Congruence of diagnoses 2 years after a first-admission diagnosis of psychosis. Arch Gen Psychiatry 2000;57:593-600.

8. Song J, Bergen SE, Kuja-Halkola R, et al. Bipolar disorder and its relation to major psychiatric disorders: a family-based study in the Swedish population. Bipolar Disord 2015;17:184-93.

9. Cardno AG, Rijsdijk FV, Sham PC, et al. A twin study of genetic relationships between psychotic symptoms. Am J Psychiatry 2002; 159:539-45.

10. Nordsletten AE, Larsson H, Crowley JJ, et al. Patterns of nonrandom mating within and across 11 major psychiatric disorders. JAMA Psychiatry 2016;73:354-61.

11. Duffy A, Alda M, Kutcher S, et al. A prospective study of the offspring of bipolar parents responsive and nonresponsive to lithium treatment. J Clin Psychiatry 2002;63:1171-8.

12. Manchia M, Cullis J, Turecki G, et al. The impact of phenotypic and genetic heterogeneity on results of genome wide association studies of complex diseases. PLoS ONE 2013;8:e76295.

13. Robins E, Guze SB. Establishment of diagnostic validity in psychiatric illness: its application to schizophrenia. Am J Psychiatry 1970; 126:983-7.

14. Franchini L, Serretti A, Gasperini M, et al. Familial concordance of fluvoxamine response as a tool for differentiating mood disorder pedigrees. J Psychiatr Res 1998;32:255-9.

15. Grof P, Duffy A, Cavazzoni P, et al. Is response to prophylactic lithium a familial trait? J Clin Psychiatry 2002;63:942-7.

\section{We believe in open access to research}

To ensure continued worldwide free access to all JPN content, Research and Review articles accepted for publication are subject to an article processing fee of $\$ 1750$ (Canadian funds), payable on acceptance.

\section{Benefits of open access}

- For researchers and institutions: increased visibility, usage and impact for their work

- For government: a better return on investment for funding research

- For society: efficient, effective patient care resulting in better outcomes

JPN has an impact factor of 5.16 (2016 ISI data), making it the highest-ranking open access journal in both the psychiatry and neuroscience categories. JPN articles are available free of charge on the journal website (jpn.ca) and in PubMed Central. 